\title{
NUMERICAL APPROXIMATION OF SOLUTION DERIVATIVES OF SINGULARLY PERTURBED PARABOLIC PROBLEMS OF CONVECTION-DIFFUSION TYPE
}

\author{
J. L. GRACIA AND E. O'RIORDAN
}

\begin{abstract}
Numerical approximations to the solution of a linear singularly perturbed parabolic convection-diffusion problem are generated using a backward Euler method in time and an upwinded finite difference operator in space on a piecewise-uniform Shishkin mesh. A proof is given to show first order convergence of these numerical approximations in an appropriately weighted $C^{1}$-norm. Numerical results are given to illustrate the theoretical error bounds.
\end{abstract}

\section{INTRODUCTION}

The solutions of singularly perturbed problems typically contain steep gradients in narrow regions of the domain, often referred to as layer regions. Layer adapted meshes, such as piecewise-uniform Shishkin meshes [5] or Bakhvalov meshes [4, have been designed to concentrate a significant proportion of the mesh points into these layer regions and thereby generate pointwise globally accurate piecewisepolynomial approximations to the continuous solution, irrespective of the size of the singular perturbation parameter. An additional feature of these layer-adapted meshes is that accurate approximations to the first derivative of the solution can be easily generated. For ease of reference, we shall refer to this additional feature of layer-adapted meshes as flux-capturing. In this paper, we present a proof of this flux-capturing property of Shishkin meshes in the case of a singularly perturbed parabolic problem.

When estimating the error in a numerical approximation, relative errors are more relevant than absolute errors. In many cases, the continuous solution is initially normalized to have a maximum value of $O(1)$, and then a pointwise bound in the maximum norm on the absolute error is equivalent to a bound on the relative error, measured in the maximum norm. In the context of singularly perturbed problems, these comments are pertinent, as there are different scales involved in the problem. In particular, the magnitude of the derivative can vary significantly within the layer regions as compared to its behavior outside the layer regions. For this reason, the appropriate norm to measure the error in approximating the flux needs to be examined closely.

Given that the singularities appearing in the solution of singularly perturbed problems are pointwise singularities, it is natural [5] to employ pointwise norms to

Received by the editor February 19, 2013 and, in revised form, September 24, 2014.

2010 Mathematics Subject Classification. Primary 65M15, 65M12.

Key words and phrases. Singular perturbation, approximation of derivatives, convectiondiffusion problem, Shishkin mesh.

The second author is the corresponding author. 
measure accuracy. Below we will discuss the following discrete versions of $C^{0}, C^{1}$ and weighted- $C^{1}$-norms, defined over a finite set of mesh points $\Omega^{N}:=\left\{x_{i}\right\}_{i=0}^{N}$ :

$$
\begin{aligned}
\|u\|_{\Omega^{N}} & :=\max _{x_{i} \in \Omega^{N}}\left|u\left(x_{i}\right)\right|, \\
\|u\|_{1, \Omega^{N}} & :=\left\|D^{-} u\right\|_{\Omega^{N} \backslash\left\{x_{0}\right\}}+\|u\|_{\Omega^{N}}, \\
\|u\|_{1, w, \Omega^{N}} & :=\left\|w D^{-} u\right\|_{\Omega^{N} \backslash\left\{x_{0}\right\}}+\|u\|_{\Omega^{N}},
\end{aligned}
$$

where $D^{-} u$ is the discrete backward derivative defined by

$$
D^{-} u\left(x_{i}\right):=\frac{u\left(x_{i}\right)-u\left(x_{i-1}\right)}{x_{i}-x_{i-1}} .
$$

However, the value of a nodal error estimate depends on the choice of mesh points. Global accuracy over the entire domain is a more neutral measure. Hence, we will consider the merits of various weighted $-C^{1}$-norms defined over a measurable region $R$ as follows:

$$
\|u\|_{1, w, R}:=\left\|w u_{x}\right\|_{R}+\|u\|_{R}, \quad\|u\|_{R}:=\operatorname{ess} \sup _{x \in R}|u(x)| .
$$

For a singularly perturbed boundary value problem of the form

$$
\varepsilon u^{\prime \prime}+a u^{\prime}=f, x \in \Omega:=(0,1) ; u(0)=A, u(1)=B ; \quad a(x) \geq \alpha>0,
$$

it was established in [5] that for a numerical solution $U^{N}$ generated using a standard upwind finite difference operator and an appropriate piecewise-uniform Shishkin mesh, one has a global error bound of the form

$$
\|u-\bar{U}\|_{1, \varepsilon, \Omega}:=\varepsilon\left\|u^{\prime}-(\bar{U})^{\prime}\right\|_{\Omega}+\|u-\bar{U}\|_{\Omega} \leq C N^{-1} \ln N,
$$

where $\bar{U}$ denotes a piecewise linear interpolant over the domain $\bar{\Omega}$ of the discrete solution $U^{N}$. Throughout this paper, $C$ denotes a generic constant that is independent of the singular perturbation parameter $\varepsilon$ and of all discretization parameters. That is, the bound in (2) states that the numerical method is parameter-uniform [5] in the $\varepsilon$-weighted norm $\|\cdot\|_{1, \varepsilon, \Omega}$.

Gartland [7] measured the errors from an exponentially fitted compact finite difference operator on a locally quasi-uniform exponentially graded mesh in a discrete version of this $\varepsilon$-weighted norm $\|\cdot\|_{1, \varepsilon, \Omega}$. However, the number of mesh points required in the Gartland mesh depends (albeit logarithmically) on $1 / \varepsilon$. Moreover, in the context of parameter-uniform numerical methods [5], exponentially fitted finite difference schemes (which are designed to be nodally exact in the case of constant coefficients) are limited to certain classes of singularly perturbed problems. Andreev [1] presented sharp bounds on the continuous solution measured in $\|\cdot\|_{1, \varepsilon, \Omega}$ and the discrete solution of a monotone three point difference scheme on arbitrary non-uniform grids in a discrete version of the norm $\|\cdot\|_{1, \varepsilon, \Omega}$ [2]. These results can be used to derive parameter-uniform global error bounds in $\|\cdot\|_{1, \varepsilon, \Omega}$ in the case of problem (11).

Note that the error in the estimate of the derivative term in (2) has been normalized by the factor $\varepsilon$, as $\varepsilon\left\|u^{\prime}\right\|_{\Omega}=O(1)$. However, the derivatives of the solution only require scaling within the layer. For example, in the case of problem (1), we note that

$$
\left|u^{\prime}(x)\right| \leq C, \quad x \geq C \varepsilon \ln (1 / \varepsilon) .
$$

Hence the scaling by the factor $\varepsilon$ in the error bound (2) is not appropriate if this error bound is restricted to points outside the layer region $[0, C \varepsilon \ln (1 / \varepsilon)]$. 
In [12] Kopteva and Stynes established an error estimate of the form

$$
\begin{array}{r}
\varepsilon\left|u^{\prime}\left(x_{i-0.5}\right)-D U\left(x_{i}\right)\right| \leq C N^{-1} \ln N, \quad x_{i} \leq C \varepsilon \ln N, \\
\left|u^{\prime}\left(x_{i-0.5}\right)-D U\left(x_{i}\right)\right| \leq C N^{-1} \ln N, \quad x_{i} \geq C \varepsilon \ln N
\end{array}
$$

(where $D U$ denotes a discrete derivative of $U$ ) for Shishkin and (corresponding bounds) for Bakhvalov meshes. The bound outside the computational layer region $[0, C \varepsilon \ln N]$ is now an unweighted $C^{1}$ error bound.

In the context of nodal accuracy on a certain mesh $\Omega^{N}$, the discrete weighted norm

$$
\|u\|_{1, z, \Omega^{N}}:=\left\|z D^{-} u\right\|_{\Omega^{N}}+\|u\|_{\Omega^{N}}, \quad z\left(x_{i}\right):=\left\{\begin{array}{l}
\varepsilon, \text { if } \alpha x_{i} \leq \varepsilon \ln N \\
1, \text { if } \alpha x_{i}>\varepsilon \ln N
\end{array}\right.
$$

appears to be a reasonable discrete norm to use to measure accuracy in the approximating solutions of singularly perturbed problems. However, observe that in the classical case of $N^{-1} \leq \varepsilon$, the scaling factor of $\varepsilon$ for mesh points within the region $(\varepsilon \ln (1 / \varepsilon), \varepsilon \ln N)$ is not appropriate.

It is also worth remarking that, in the case of singularly perturbed ordinary differential equations, if a scheme is nodally second order (ignoring logarithmic factors) in $\|\cdot\|_{\Omega^{N}}$ on a Shishkin mesh, then it is nodally first order in the $\varepsilon$-weighted $C^{1}$-norm $\|\cdot\|_{1, \varepsilon, \Omega^{N}}$. In particular, Andreev and Savin 3 analysed a modification of Samarskii's monotone finite difference operator on a piecewise-uniform mesh to establish an error bound in $\|\cdot\|_{\Omega^{N}}$ of the form $C\left(N^{-1} \ln N\right)^{2}$, and thereby one has an error bound of the form $C N^{-1} \ln N$ in the discrete norm $\|\cdot\|_{1, \varepsilon, \Omega^{N}}$ for the scheme presented in [3].

In this paper, we confine our attention to a simple finite difference scheme on a standard piecewise-uniform Shishkin mesh as it applies to a singularly perturbed partial differential equation defined over a region $G:=\Omega \times(0, T]$, which is a timedependent version of problem (1). More sophisticated finite difference operators on various layer-adapted meshes, which are second order in space and first order in time, exist in the literature. However, in contrast to the case of an ordinary differential equation, one cannot directly deduce a first order error bound in the discrete norm

$$
\|u\|_{1, \varepsilon, G^{N, M}}:=\varepsilon\left\|D_{x}^{-} u\right\|_{\bar{G}^{N, M} \backslash\left\{\left(x_{0}, t_{j}\right)\right\}_{j=0}^{M}}+\left\|D_{t}^{-} u\right\|_{\bar{G}^{N, M} \backslash\left\{\left(x_{i}, t_{0}\right)\right\}_{i=0}^{N}}+\|u\|_{\bar{G}^{N, M}},
$$

from such nodal error bounds. Kopteva [11] analysed a non-monotone finite difference scheme on a Shishkin mesh, which is second order in both space and time; thereby, this scheme is first order in the discrete norm $\|\cdot\|_{1, \varepsilon, G^{N}}$ (assuming $M=C N$ ). In this paper, we choose to establish convergence in a global norm (specified below) for a monotone finite difference scheme, which is only first order in both space and time.

In the case of singularly perturbed parabolic problems, Shishkin [15] introduced a sophisticated global metric which is designed to measure the pointwise relative error in estimating the first derivative both within and outside the layer region. In the case of the time dependent version of problem (11), this new weighted metric is, in essence, of the form

$$
\|v\|_{1, s, G}:=\left\|s v_{x}\right\|_{G}+\left\|v_{t}\right\|_{G}+\|v\|_{G}, \quad s(x, t):=\frac{\varepsilon}{\varepsilon+e^{-a\left(x_{1}, t\right) x / \varepsilon}},
$$


where $x_{1}$ is the first internal mesh point in the spatial direction. In [15], Shishkin shows that the error bound (2) applies in the case of upwinding on a piecewiseuniform mesh, but the same numerical scheme is not $\varepsilon$-uniformly convergent in this new metric. Conditions can be imposed on the parameters in a generalized piecewise-uniform Shishkin mesh (see [15, §6] for details) so that the numerical approximations converge almost $\varepsilon$-uniformly in this metric $\|\cdot\|_{1, s, G}$, to be precise, at a rate of $O\left(\varepsilon^{-\nu} N^{-1}\right)$, where $\nu>0$ is arbitrarily small. We refer the reader to [15] for further details. Shishkin extended these ideas on suitable metrics to the case of singularly perturbed elliptic partial differential equations in [14].

In this paper, we choose the simpler (but cruder) global metric of simply scaling the first derivative by the constant $\varepsilon$ within the layer and using no scaling factor outside the layer. Hence, instead of $\|\cdot\|_{1, s, G}$ we will measure the errors in the following weighted $C^{1}$-norm:

$$
\begin{aligned}
\|v\|_{1, \chi, G} & :=\left\|\chi v_{x}\right\|_{G}+\left\|v_{t}\right\|_{G}+\|v\|_{G}, \\
\chi(x) & :=\left\{\begin{array}{l}
\varepsilon, \text { if } \alpha|x-p| \leq 2 \varepsilon \ln (1 / \varepsilon), \\
1, \text { if } \alpha|x-p|>2 \varepsilon \ln (1 / \varepsilon),
\end{array}\right.
\end{aligned}
$$

where $p=0$ or $p=1$ depending on the location of the boundary layer. In this paper, we examine a problem with the boundary layer located on the right (where $p=1)$. Note that the weighting function $\chi(x)$ is excessive in the region where $C \varepsilon \ll \alpha|x-p| \leq 2 \varepsilon \ln (1 / \varepsilon)$.

In $\S 2$ the continuous problem is stated and parameter-explicit bounds on the derivatives of the solution are established by decomposing the solution into regular and singular components. In $\S 3$ the numerical method is described and appropriate bounds on the nodal errors are given. These estimates are used in $\S 4$ and $\S 5$ to establish scaled nodal error bounds on approximations of the space and time derivatives, respectively. In $\S 6$ the main result of the paper, which establishes an error estimate in the norm $\|\cdot\|_{1, \chi, G}$, is given in Theorem 7 . Some numerical results are given in the final section of the paper.

\section{Continuous problem}

Consider the following class of singularly perturbed parabolic problems:

$$
L_{\varepsilon} u:=-\varepsilon u_{x x}+a(x, t) u_{x}+b(x, t) u+c(t) u_{t}=f(x, t), \text { in } G:=\Omega \times(0, T],
$$

(4b) $u=0, \quad$ on $\Gamma_{B} \cup \Gamma_{L} \cup \Gamma_{R}, \quad 0<\varepsilon \leq 1 ; \quad a(x, t)>\alpha>0, c(t) \geq c_{0}>0$,

(4c) $b(x, t) \geq \max \left\{\left\|a_{x}\right\|_{G},\left\|a_{t}\right\|_{G},\left\|c_{t}\right\|_{G}\right\}+\beta, \beta>0$,

where $\Omega:=(0,1), \Gamma_{B}:=\{(x, 0) \mid 0 \leq x \leq 1\}, \Gamma_{L}:=\{(0, t) \mid 0 \leq t \leq T\}, \Gamma_{R}:=$ $\{(1, t) \mid 0 \leq t \leq T\}$ and $\Gamma:=\bar{G} \backslash G$. Since the problem is linear, there is no loss in generality in assuming zero boundary/initial conditions. The constraint (4:) on the coefficient $b(x, t)$ can be transferred to the time variable by using the change of variable $u=v e^{\gamma t}$, where $\gamma>0$ is sufficiently large. We assume that the data of the problem satisfy regularity and compatibility conditions so that the solution of 
problem (4) is such that $u \in C^{6+\gamma}(\bar{G}) 1$ (see [6] and [13]) and so that the analysis presented below is applicable.

It is well-known that the differential operator associated with (4) satisfies a comparison principle. From this, one can establish the stability estimate

$$
|u(x, t)| \leq \min \left\{\frac{x}{\alpha}, \frac{t}{c_{0}}\right\}\|f\|_{G} .
$$

Motivated by the bounds given in 9 and 15] we present the following bounds on the derivatives of the regular and singular components of $u$. For the sake of completeness, we outline a proof of these bounds here.

Theorem 1. The solution of (4) can be written in the form $u=v+w$, where the regular component $v \in C^{6+\gamma}(\bar{G})$ satisfies

$$
L_{\varepsilon} v=f, \text { in } G ; \quad v=u \text {, on } \Gamma_{B} \cup \Gamma_{L},
$$

and $v=v^{*}$ can be specified on the boundary $\Gamma_{R}$ so that

$$
\left\|\frac{\partial^{k+m} v}{\partial x^{k} \partial t^{m}}\right\|_{G} \leq C\left(1+\varepsilon^{3-k-m}\right), \quad 0 \leq k+2 m \leq 6 .
$$

The singular component $w$ satisfies the homogeneous differential equation

$$
L_{\varepsilon} w=0 \text {, in } G ; \quad w=0 \text {, on } \Gamma_{B} \cup \Gamma_{L} ; w=u-v \text {, on } \Gamma_{R},
$$

and for all points $(x, t) \in \bar{G}$ its derivatives satisfy the pointwise bounds

$$
\left|\frac{\partial^{k+m} w}{\partial x^{k} \partial t^{m}}(x, t)\right| \leq C \varepsilon^{-k}\left(1+\varepsilon^{2-m}\right) e^{-\alpha(1-x) / \varepsilon}, \quad 0 \leq k+2 m \leq 6 .
$$

Proof. Using the stretched variables $\zeta:=(1-x) / \varepsilon, \eta:=t / \varepsilon$ and the a priori bounds [13, pg. 320, Theorem 5.2], one deduces the bounds

$$
\left\|\frac{\partial^{k+m} u}{\partial x^{k} \partial t^{m}}\right\|_{G} \leq C \varepsilon^{-k-m}, \quad 0 \leq k+2 m \leq 6 .
$$

Consider the extended domain $G^{*}:=\{(0, A) \times(0, B) ; A>1, B>T\}$, with associated extended boundaries $\Gamma_{L}^{*}, \Gamma_{B}^{*}$ and $a^{*}, b^{*}, c^{*}, f^{*}$ are smooth extensions of $a, b, c, f$ to the extended domain $G^{*}$. The first order reduced operator $L_{0}^{*}$ is defined by

$$
L_{0}^{*} z:=a^{*} z_{x}+b^{*} z+c^{*} z_{t}, \quad \text { in } \bar{G}^{*} \backslash\left(\Gamma_{B}^{*} \cup \Gamma_{L}^{*}\right), \quad z=z, \text { on } \Gamma_{B}^{*} \cup \Gamma_{L}^{*} .
$$

The regular component $v$ is composed of the reduced solution $v_{0}$, higher order terms $v_{1}, v_{2}$ in an asymptotic expansion and a remainder term $R$, given by

$$
\begin{aligned}
v^{*} & =v_{0}^{*}+\varepsilon v_{1}^{*}+\varepsilon^{2} v_{2}^{*}+\varepsilon^{3} R^{*} ; \\
L_{0}^{*} v_{0}^{*} & =f^{*}, \quad \text { in } G^{*}, \quad v_{0}^{*}=u, \text { on } \Gamma_{B}^{*} \cup \Gamma_{L}^{*} ; \\
L_{0}^{*} v_{i}^{*} & =\left(v_{i-1}^{*}\right)_{x x}, \text { in } G^{*}, v_{i}^{*}=0, \text { on } \Gamma_{B}^{*} \cup \Gamma_{L}^{*}, \quad i=1,2 ; \\
L_{\varepsilon}^{*} R^{*} & =\left(v_{2}^{*}\right)_{x x}, \text { in } G^{*}, \quad R^{*}=0, \text { on } \bar{G}^{*} \backslash G^{*} .
\end{aligned}
$$

The bounds on the derivatives of $v^{*}$ (and hence $v$ ) are then easily deduced.

The singular component $w$ can be decomposed as follows:

$$
w(x, t)=(u-v)(1, t) \Psi(x, t)+\varepsilon R(x, t),
$$

\footnotetext{
${ }^{1}$ The space $C^{n+\gamma}(D)$ is the set of all functions whose derivatives of order $n$ are Hölder continuous of degree $\gamma>0$. That is,

$$
C^{n+\gamma}(D):=\left\{z: \frac{\partial^{i+j} z}{\partial x^{i} \partial t^{j}} \in C^{\gamma}(D), 0 \leq i+2 j \leq n\right\}
$$
}


where, for each value of $t$, the unit boundary layer function $\Psi$ satisfies

$$
-\varepsilon \Psi_{x x}+a(x, t) \Psi_{x}=0, \quad \Psi(0, t)=0, \Psi(1, t)=1 .
$$

Note that

$$
\Psi(x, t)=\frac{\int_{r=0}^{x} e^{-\int_{s=r}^{1} \frac{a(s, t)}{\varepsilon} d s} d r}{\int_{r=0}^{1} e^{-\int_{s=r}^{1} \frac{a(s, t)}{\varepsilon} d s} d r} .
$$

Using the strict inequality $a>\alpha$ and $((1-\theta) t)^{m} e^{-t} \leq m ! e^{-\theta t}, 0<\theta<1, t \geq 0$, we have that

$$
\left|\frac{\partial^{m} \Psi(x, t)}{\partial t^{m}}\right| \leq C e^{-\alpha(1-x) / \varepsilon}, \quad 0 \leq m \leq 3 .
$$

For the remainder term, $R(x, t)=0,(x, t) \in \Gamma$ and for all $(x, t) \in G$,

$$
\varepsilon L_{\varepsilon} R=-(u-v)(1, t) b(x, t) \Psi(x, t)-c(t)((u-v)(1, t) \Psi(x, t))_{t} .
$$

Hence

$$
|R(x, t)| \leq C e^{-\alpha(1-x) / \varepsilon} .
$$

Using the stretched variables and the localized bounds on the derivatives [13, pg. 352, (10.5)] one can deduce the bounds

$$
\left|\frac{\partial^{k+m} R(x, t)}{\partial x^{k} \partial t^{m}}\right| \leq C \varepsilon^{-k-m} e^{-\alpha(1-x) / \varepsilon}, \quad 0 \leq k+2 m \leq 6 .
$$

Hence,

$$
\left|\frac{\partial^{k+m} w(x, t)}{\partial x^{k} \partial t^{m}}\right| \leq C\left(1+\varepsilon^{1-m}\right) \varepsilon^{-k} e^{-\alpha(1-x) / \varepsilon}, \quad 0 \leq k+2 m \leq 6 .
$$

We improve the above bounds on the time derivatives by noting that for $m=1,2,3$,

$$
\begin{aligned}
\left|L_{\varepsilon} \frac{\partial^{m} w(x, t)}{\partial t^{m}}\right| \leq & C \sum_{j=0}^{m-1}\left|\frac{\partial^{j} w(x, t)}{\partial t^{j}}\right|+C \sum_{j=0}^{m-1}\left|\frac{\partial^{j+1} w(x, t)}{\partial t^{j} \partial x}\right|,(x, t) \in G, \\
& \frac{\partial^{m} w(x, t)}{\partial t^{m}}=0,(x, t) \in \Gamma_{B} \cup \Gamma_{L}, \quad\left|\frac{\partial^{m} w(1, t)}{\partial t^{m}}\right| \leq C,
\end{aligned}
$$

which implies that

$$
\left|\frac{\partial^{m} w(x, t)}{\partial t^{m}}\right| \leq C\left(1+\varepsilon^{2-m}\right) e^{-\alpha(1-x) / \varepsilon}, \quad 1 \leq m \leq 3 .
$$

From the equation $\left(L_{\varepsilon} w\right)_{t t}=0$ we have that, for all $t \geq 0$,

$$
\begin{gathered}
-\varepsilon \frac{\partial^{2}}{\partial x^{2}} w_{t t}+a(x, t) \frac{\partial}{\partial x} w_{t t}=g(x, t), x \in(0,1), \quad w_{t t}(0, t)=0,\left|w_{t t}(1, t)\right| \leq C, \\
|g(x, t)| \leq C \sum_{j=0}^{3}\left|\frac{\partial^{j} w(x, t)}{\partial t^{j}}\right|+C \sum_{j=0}^{1}\left|\frac{\partial^{j+1} w(x, t)}{\partial t^{j} \partial x}\right| \leq C\left(1+\varepsilon^{-1}\right) e^{-\alpha(1-x) / \varepsilon} .
\end{gathered}
$$

For each time $t$, we use this boundary value problem for $w_{t t}$ to deduce (use argument from [5, pp. 46-47] with $x \rightarrow 1-x$ ) that

$$
\left|\frac{\partial^{i+2}}{\partial x^{i} \partial t^{2}} w(x, t)\right| \leq C\left(1+\varepsilon^{-i}\right) e^{-\alpha(1-x) / \varepsilon}, \quad i=1,2 .
$$

Collecting all these bounds together completes the proof.

Remark 1. The proof of the bounds in Theorem 1 simplifies significantly in the special case of $a(x)$ being independent of time. In fact, in this particular case, all the time derivatives of the solution $u$ of (4) are $\varepsilon$-uniformly bounded. 


\section{Numerical SCHEME}

Consider a uniform mesh in time $\bar{\omega}^{M}=\{k \tau, 0 \leq k \leq M, \tau=T / M\}$ and a piecewise-uniform Shishkin mesh $\bar{\Omega}^{N}$ [5] in space on which numerical approximations of the solution of problem (4) are generated. The subintervals $[0,1-\sigma]$, and $[1-\sigma, 1]$ are each uniformly subdivided into $N / 2$ mesh intervals, where the transition parameter $\sigma$ is defined by

$$
\sigma:=\min \left\{\frac{1}{2}, 2 \frac{\varepsilon}{\alpha} \ln N\right\} .
$$

Then, the grid in the space variable $\bar{\Omega}^{N}=\left\{x_{i}\right\}$ is given by

$$
x_{i}= \begin{cases}i H, & \text { if } \quad 0 \leq i \leq N / 2, \\ (1-\sigma)+(i-N / 2) h, & \text { if } \quad N / 2 \leq i \leq N,\end{cases}
$$

where the step sizes are $h:=2 \sigma / N$ and $H:=2(1-\sigma) / N$. We denote the local step sizes by $h_{j}:=x_{j}-x_{j-1}$ for $j=1, \ldots, N$, and we define the following sets of mesh points:

$$
\bar{G}^{N, M}:=\bar{\Omega}^{N} \times \bar{\omega}^{M}, G^{N, M}:=\bar{G}^{N, M} \cap G, \quad \Gamma^{N, M}:=\bar{G}^{N, M} \backslash G^{N, M} .
$$

We combine this mesh with a simple fully implicit finite difference operator, which uses the classical upwind approximation for the space derivatives, to produce the finite difference method:

(9)

$$
\left\{\begin{array}{l}
L^{N, M} U\left(x_{i}, t_{j}\right)=f\left(x_{i}, t_{j}\right),\left(x_{i}, t_{j}\right) \in G^{N, M}, U\left(x_{i}, t_{j}\right)=0,\left(x_{i}, t_{j}\right) \in \Gamma^{N, M}, \\
L^{N, M} U\left(x_{i}, t_{j}\right):=\left(-\varepsilon \delta_{x}^{2}+a D_{x}^{-}+b I+c D_{t}^{-}\right) U\left(x_{i}, t_{j}\right),
\end{array}\right.
$$

where

$$
\begin{array}{r}
U_{i, j}:=U\left(x_{i}, t_{j}\right), D_{t}^{-} U_{i, j}:=\frac{U_{i, j}-U_{i, j-1}}{\tau}, \delta_{x}^{2} U_{i, j}:=\frac{1}{\hbar_{i}}\left(D_{x}^{+} U_{i, j}-D_{x}^{-} U_{i, j}\right), \\
D_{x}^{+} U_{i, j}:=\frac{U_{i+1, j}-U_{i, j}}{h_{i+1}}, D_{x}^{-} U_{i, j}:=\frac{U_{i, j}-U_{i-1, j}}{h_{i}}, \hbar_{i}:=\frac{h_{i}+h_{i+1}}{2} .
\end{array}
$$

Throughout the analysis in this paper we assume that

$$
\sigma=2 \frac{\varepsilon}{\alpha} \ln N, \quad C_{1} N \leq M \leq C_{2} N .
$$

It is well-known that the finite difference operator associated with problem (9) satisfies a discrete comparison principle. To obtain appropriate bounds of the error in the maximum norm, consider the following decomposition of the numerical solution $U=V+W$, where the discrete regular $V$ and singular $W$ components satisfy the problems

$$
L^{N, M} V=f,\left.V\right|_{\Gamma^{N, M}}=\left.v\right|_{\Gamma^{N, M}}, \quad L^{N, M} W=0,\left.W\right|_{\Gamma^{N, M}}=\left.w\right|_{\Gamma^{N, M}} .
$$

In the next theorem we establish bounds on the error associated with the regular and singular components, which are used later in the error analysis in the weighted $C^{1}$-norm $\|\cdot\|_{1, \chi, G}$.

Theorem 2. Assume (10). For all $t_{j} \geq 0$, we have the following bounds:

$$
\begin{array}{ll}
\left|(V-v)\left(x_{i}, t_{j}\right)\right| \leq C t_{j} N^{-1}, & \text { if } x_{i} \in[0,1], \\
\left|(W-w)\left(x_{i}, t_{j}\right)\right| \leq C N^{-2}, & \text { if } x_{i} \in[0,1-\sigma], \\
\left|(W-w)\left(x_{i}, t_{j}\right)\right| \leq C N^{-2}+C t_{j} N^{-1} \ln N, & \text { if } x_{i} \in(1-\sigma, 1],
\end{array}
$$

where $v, w$ are the solutions of problems (5a), (6- a) and $V, W$ are defined in (11). 
Proof. From the truncation error bound $\left\|L^{N, M}(V-v)\right\|_{\bar{G}^{N, M}} \leq C N^{-1}$ and the discrete maximum principle one has the nodal error bound

$$
\left|(V-v)\left(x_{i}, t_{j}\right)\right| \leq C t_{j} N^{-1} \text {. }
$$

For the singular component, we distinguish two cases depending on the location of the grid point. First, outside the layer, we have that [5]

$$
\left|(W-w)\left(x_{i}, t_{j}\right)\right| \leq\left|W\left(x_{i}, t_{j}\right)\right|+\left|w\left(x_{i}, t_{j}\right)\right| \leq C N^{-2}, \quad x_{i} \leq 1-\sigma .
$$

If $x_{i} \in(1-\sigma, 1)$, then the truncation error satisfies

$$
\left|L^{N, M}(w-W)\left(x_{i}, t_{j}\right)\right| \leq C\left(\tau+h\left|w_{x x}\left(x_{i}^{*}, t_{j}\right)\right|+\varepsilon h\left|w_{x x x}\left(x_{i}^{* *}, t_{j}\right)\right|\right),
$$

with $x_{i}^{*} \in\left(x_{i}-h, x_{i}\right)$, and $x_{i}^{* *} \in\left(x_{i}-h, x_{i}+h\right)$. Using the inequalities

$$
\begin{aligned}
&\left|w_{x x}\left(x_{i}^{*}, t_{j}\right)\right|=\left|\int_{0}^{t_{j}} w_{x x t}\left(x_{i}^{*}, s\right) d s\right| \leq C t_{j} \varepsilon^{-2} e^{-\alpha\left(1-x_{i}\right) / \varepsilon}, \\
&\left|w_{x x x}\left(x_{i}^{* *}, t_{j}\right)\right| \leq C t_{j} \varepsilon^{-3} e^{-\alpha\left(1-x_{i+1}\right) / \varepsilon}
\end{aligned}
$$

within the layer region, we obtain the truncation error bound

$$
\left|L^{N, M}(w-W)\left(x_{i}, t_{j}\right)\right| \leq C\left(\tau+\frac{N^{-1} \ln N}{\varepsilon} t_{j} e^{-\alpha\left(1-x_{i+1}\right) / \varepsilon}\right), 1-\sigma<x_{i}<1 .
$$

Use the discrete barrier function

$$
C\left(t_{j} N^{-1} \ln N(1+\alpha(h / \varepsilon))^{i+2-N}+\tau t_{j}+C N^{-2}\right)
$$

and $\left|(w-W)\left(1-\sigma, t_{j}\right)\right| \leq C N^{-2}$ to complete the proof.

\section{Nodal approximation of SPACE DERIVATiVes}

Consistency and stability form a classical argument in numerical analysis, which is typically employed to deduce a nodal error bound. To bound the quantity $D_{x}^{-}(U-u)$ at the mesh points, we use an argument of this type by employing a bound on a quantity of the form $\left\|\hat{L}^{N, M}\left(D_{x}^{-}(U-u)\right)\right\|$, where the finite difference operator $\hat{L}^{N, M}$ is monotone and is defined below in (13).

We denote the nodal error by $e\left(x_{i}, t_{j}\right):=U\left(x_{i}, t_{j}\right)-u\left(x_{i}, t_{j}\right)$, and the associated truncation error by $\mathscr{T}\left(x_{i}, t_{j}\right):=L^{N, M} e\left(x_{i}, t_{j}\right)$. We define the discrete error flux to be

$$
\mathscr{U}_{i, j}^{-}:=D_{x}^{-} e\left(x_{i}, t_{j}\right), \quad \text { if } 0<x_{i} \leq 1 .
$$

The main purpose of this section is to deduce suitable bounds on $\mathscr{U}^{-}$.

We identify a discrete problem associated with the error flux defined over the region

$$
G_{H}^{N, M}:=G^{N, M} \cap\{(H, 1) \times(0, T]\}, \quad \bar{G}_{H}^{N, M}:=\bar{G}^{N, M} \cap\{[H, 1] \times[0, T]\} .
$$

We define a new finite difference operator $\hat{\delta}_{x}^{2}$ by

$$
\hat{\delta}_{x}^{2} Z_{i, j}:=\frac{1}{\hbar_{i}}\left(\frac{h_{i+1}}{h_{i}} D_{x}^{+}-\frac{\hbar_{i}}{\hbar_{i-1}} D_{x}^{-}\right) Z_{i, j},
$$

which has the property that

$$
\hat{\delta}_{x}^{2} D_{x}^{-} Z_{i, j} \equiv D_{x}^{-} \delta_{x}^{2} Z_{i, j}
$$

Note the following identity:

$$
D_{x}^{-}\left(P_{i, j} Q_{i, j}\right) \equiv P_{i, j} D_{x}^{-} Q_{i, j}+Q_{i-1, j} D_{x}^{-} P_{i, j} .
$$


Using these identities and $D_{x}^{-}\left(L^{N, M} e\left(x_{i}, t_{j}\right)\right)=D_{x}^{-} \mathscr{T}\left(x_{i}, t_{j}\right)$, we see that for all mesh points within the region $\bar{G}_{H}^{N, M}$, the quantity $\mathscr{U}_{i, j}^{-}$satisfies

$$
\hat{L}^{N, M} \mathscr{U}_{i, j}^{-}=D_{x}^{-} \mathscr{T}\left(x_{i}, t_{j}\right)-e\left(x_{i-1}, t_{j}\right) D_{x}^{-} b\left(x_{i}, t_{j}\right),\left(x_{i}, t_{j}\right) \in G_{H}^{N, M},
$$

where for the internal points $\left(x_{i}, t_{j}\right) \in G_{H}^{N, M}$,

$$
\hat{L}^{N, M} Z_{i, j}:=\left(-\varepsilon \hat{\delta}_{x}^{2}+a\left(x_{i-1}, t_{j}\right) D_{x}^{-}+\left(b+D_{x}^{-} a\right)\left(x_{i}, t_{j}\right) I+c\left(t_{j}\right) D_{t}^{-}\right) Z_{i, j},
$$

and $\hat{L}^{N, M} Z_{i, j}:=Z_{i, j}$ for $\left(x_{i}, t_{j}\right) \in \bar{G}_{H}^{N, M} \backslash G_{H}^{N, M}$.

Remark 2. When bounding the term $D_{x}^{-} \mathscr{T}\left(x_{i}, t_{j}\right)$ we will make use of the following truncation error bounds:

$$
\begin{aligned}
\left|D_{x}^{-}\left(u_{t}-D_{t}^{-} u\right)\left(x_{i}, t_{j}\right)\right| & =\frac{1}{\tau}\left|\int_{t=t_{j-1}}^{t_{j}} \int_{s=r}^{t_{j}} D_{x}^{-} u_{t t}\left(x_{i}, s\right) d s d r\right| \\
& \leq C \tau\left\|u_{t t x}\right\|_{\left(x_{i-1}, x_{i}\right) \times\left(t_{j-1}, t_{j}\right)} .
\end{aligned}
$$

If $h_{i-1}=h_{i}$, then

$$
\left|D_{x}^{-}\left(u_{x}-D_{x}^{-} u\right)\left(x_{i}, t_{j}\right)\right| \leq C h_{i}\left\|u_{x x x}\left(x, t_{j}\right)\right\|_{x \in\left(x_{i-2}, x_{i}\right)},
$$

and if $h_{i-1}=h_{i}=h_{i+1}$, then

$$
\left|D_{x}^{-}\left(u_{x x}-\delta_{x}^{2} u\right)\left(x_{i}, t_{j}\right)\right| \leq C h_{i}^{2}\left\|u_{x x x x x}\left(x, t_{j}\right)\right\|_{x \in\left(x_{i-2}, x_{i+1}\right)} .
$$

From the assumption that $\beta>\left\|a_{x}\right\|_{G}$, the discrete operator $\hat{L}^{N, M}$ satisfies a discrete comparison principle.

Now we deduce bounds on the regular $\mathscr{V}^{-}:=D_{x}^{-}(V-v)$ and the singular $\mathscr{W}^{-}:=D_{x}^{-}(W-w)$ components of the discrete error flux $\mathscr{U}^{-}$. We begin with the singular component. For the mesh points along the right hand boundary $x=1$, we will need an appropriate bound on the outgoing error flux $\left|D_{x}^{-}(W-w)\right|$. We achieve this by sharpening the nodal error bound given in Theorem 2, within the layer region, to reflect the fact that $(W-w)\left(1, t_{j}\right)=0$.

Lemma 1. Assume (10). For the solutions $w, W$ of the problems (6a) and (11), and for sufficiently large $N$,

$$
\varepsilon\left|D_{x}^{-}(W-w)\left(1, t_{j}\right)\right| \leq C N^{-1}(\ln N)^{2}, \quad t_{j} \geq 0 .
$$

Proof. For each $t_{j} \geq 0$, consider the discrete function $\psi\left(x_{i}, t_{j}\right)$ as the solution of the discrete problem

$$
-\varepsilon \delta_{x}^{2} \psi+\left(a\left(x_{i}, t_{j}\right)+\beta t_{j}\right) D_{x}^{-} \psi=0, x_{i} \in(1-\sigma, 1), \psi\left(1-\sigma, t_{j}\right)=1, \psi\left(1, t_{j}\right)=0 .
$$

Note that the mesh is uniform within $(1-\sigma, 1) \times(0, T]$. Define the discrete flux to be

$$
F_{i}^{j}:=D_{x}^{-} \psi\left(x_{i}, t_{j}\right)<0,
$$

which satisfies the inequalities

$$
h \sum_{i=N / 2+1}^{N} F_{i}^{j}=-1, \quad F_{i}^{j}=F_{N}^{j} \prod_{k=i}^{N-1}\left(1+\frac{\left(a\left(x_{k}, t_{j}\right)+\beta t_{j}\right) h}{\varepsilon}\right)^{-1}, i<N .
$$

Hence, for sufficiently large $N$,

$$
\left|D_{x}^{-} \psi\left(1, t_{j}\right)\right| \leq \frac{1-\left(1+\left(\|a\|_{G}+\beta T\right) \frac{h}{\varepsilon}\right)^{-1}}{h\left(1-\left(1+\left(\|a\|_{G}+\beta T\right) \frac{h}{\varepsilon}\right)^{-N / 2}\right)} \leq \frac{C}{\varepsilon} .
$$


Here we have used the inequality

$$
\left(1+K \frac{\ln N}{N}\right)^{N / 2}>e^{K \frac{\ln N}{2}\left(1-K \frac{\ln N}{2 N}\right)},
$$

and $N$ is sufficiently large. Note also that for $x_{i} \in(1-\sigma, 1)$, $\left(-\varepsilon \delta_{x}^{2}+\left(a\left(x_{i}, t_{j}\right)+\beta t_{j}\right) D_{x}^{-}\right) D_{t}^{-} \psi\left(x_{i}, t_{j}\right)=-\left(D_{t}^{-} a\left(x_{i}, t_{j}\right)+\beta\right) D_{x}^{-}\left(\psi\left(x_{i}, t_{j-1}\right)\right) \geq 0$, where we have used the identity (12) and $D_{t}^{-} \psi\left(1-\sigma, t_{j}\right)=D_{t}^{-} \psi\left(1, t_{j}\right)=0$. Also

$$
\left|\left(D_{t}^{-} a\left(x_{i}, t_{j}\right)+\beta\right) D_{x}^{-}\left(\psi\left(x_{i}, t_{j-1}\right)\right)\right| \leq C \varepsilon^{-1}\left(1+\frac{\alpha h}{\varepsilon}\right)^{i-N} .
$$

Using a discrete comparison principle, we deduce that

$$
D_{t}^{-} \psi\left(x_{i}, t_{j}\right) \geq 0, \quad\left|D_{t}^{-} \psi\left(x_{i}, t_{j}\right)\right| \leq C\left(1+\frac{\alpha h}{\varepsilon}\right)^{i+1-N}
$$

Now we define a barrier function to deduce appropriate bounds for $\mathscr{W}_{N, j}^{-}$. First, we note that, at each time level $t_{j}$, the grid function $x_{i}-1+\sigma \psi\left(x_{i}, t_{j}\right)$ is the solution of the problem

$$
\begin{gathered}
\left(-\varepsilon \delta_{x}^{2}+a\left(x_{i}, t_{j}\right) D_{x}^{-}\right)\left(x_{i}-1+\sigma \psi\left(x_{i}, t_{j}\right)\right) \\
=a\left(x_{i}, t_{j}\right)-\sigma \beta t_{j} D_{x}^{-} \psi\left(x_{i}, t_{j}\right), x_{i} \in(1-\sigma, 1), \\
\left(x_{i}-1+\sigma \psi\left(x_{i}, t_{j}\right)\right)_{x_{i}=1-\sigma}=\left(x_{i}-1+\sigma \psi\left(x_{i}, t_{j}\right)\right)_{x_{i}=1}=0 .
\end{gathered}
$$

So, by the discrete maximum principle $x_{i}-1+\sigma \psi\left(x_{i}, t_{j}\right) \geq 0$. Note that

$$
L^{N, M}\left(x_{i}-1+\sigma \psi\left(x_{i}, t_{j}\right)\right) \geq a\left(x_{i}, t_{j}\right)+\sigma\left(c\left(t_{j}\right) D_{t}^{-}-\beta t_{j} D_{x}^{-}\right) \psi\left(x_{i}, t_{j}\right) \geq a\left(x_{i}, t_{j}\right) .
$$

Define the discrete barrier function

$$
B_{1}\left(x_{i}, t_{j}\right):=C\left\|L^{N, M}(W-w)\right\|_{x \in(1-\sigma, 1)}\left(x_{i}-1+\sigma \psi\left(x_{i}, t_{j}\right)\right)+C N^{-2},
$$

where $L^{N, M}(W-w)$ is the truncation error associated with the singular component. Recall that in the boundary layer region

$$
\left\|L^{N, M}(W-w)\right\|_{x \in(1-\sigma, 1)} \leq C \varepsilon^{-1} N^{-1} \ln N .
$$

We then have that $\left|(W-w)\left(x_{i}, t_{j}\right)\right| \leq B_{1}\left(x_{i}, t_{j}\right)$ for $x_{i} \in[1-\sigma, 1]$. Therefore,

$$
\begin{aligned}
\varepsilon\left|\mathscr{W}_{N, j}^{-}\right| & =\frac{\varepsilon}{h}\left|(W-w)\left(1-h, t_{j}\right)\right| \\
& \leq C \varepsilon\left\|L^{N, M}(W-w)\right\|_{x \in(1-\sigma, 1)}\left(1+\sigma\left|D_{x}^{-} \psi\left(1, t_{j}\right)\right|\right)+C N^{-1} \\
& \leq C N^{-1}(\ln N)^{2}
\end{aligned}
$$

which is the required result.

Theorem 3. Assume (10). Then, for all $t_{j} \geq 0$,

$$
\begin{array}{ll}
\left|D_{x}^{-}(W-w)\left(x_{i}, t_{j}\right)\right| \leq C N^{-1}, & \text { if } x_{i} \leq 1-\sigma, \\
\varepsilon\left|D_{x}^{-}(W-w)\left(x_{i}, t_{j}\right)\right| \leq C N^{-1}(\ln N)^{2}, & \text { if } x_{i}>1-\sigma,
\end{array}
$$

where $W$ is the solution of (11) and $w$ is the solution of (6a).

Proof. Note that outside the layer region, Theorem 2 implies that

$$
\left|\mathscr{W}_{i, j}^{-}\right| \leq C N^{-1}, \quad \text { if } x_{i} \in[0,1-\sigma], t_{j} \geq 0 .
$$

Also, for $x_{i}=1-\sigma+h, 1-\sigma+2 h$,

$$
\begin{aligned}
\left|w\left(x_{i}, t_{j}\right)\right| & \leq C e^{\alpha 2 h / \varepsilon} e^{-\alpha \sigma / \varepsilon} \leq C N^{-2} \\
\left|W\left(x_{i}, t_{j}\right)\right| & \leq C(1+\alpha h / \varepsilon)^{2}(1+\alpha h / \varepsilon)^{-N / 2} \leq C N^{-2} .
\end{aligned}
$$


Hence,

$$
\varepsilon\left|\mathscr{W}_{i, j}^{-}\right| \leq C N^{-1}, x_{i}=1-\sigma+h, 1-\sigma+2 h .
$$

In the layer region $(1-\sigma+2 h, 1) \times(0, T)$ we will obtain the bounds by using (13). Initially, $\mathscr{W}_{i, 0}^{-}=0, x_{i} \in(1-\sigma, 1)$, and we established the required bound on the right boundary in (15). For $x_{i} \in(1-\sigma+2 h, 1), t_{j}>0$ and $G_{i}^{j}:=\left(x_{i-1}, x_{i}\right) \times\left(t_{j-1}, t_{j}\right)$, using (14) we get that

$$
\begin{aligned}
\left|\hat{L}^{N, M} \mathscr{W}_{i, j}^{-}\right| \leq & C \tau\left\|w_{t t x}\right\|_{G_{i}^{j}}+C h\left(\varepsilon h\left\|w_{x x x x x}\right\|_{G_{i-1}^{j} \cup G_{i}^{j} \cup G_{i+1}^{j}}\right. \\
& \left.+\left\|a_{x}\right\|_{G}\left\|w_{x x}\right\|_{G_{i}^{j}}+\|a\|_{G}\left\|w_{x x x}\right\|_{G_{i-1}^{j} \cup G_{i}^{j}}\right)+C N^{-1} \ln N \\
\leq & \frac{C}{\varepsilon} \frac{N^{-1} \ln N}{\varepsilon} e^{-\alpha\left(1-x_{i}\right) / \varepsilon}+C N^{-1} \ln N .
\end{aligned}
$$

Use the discrete barrier function (and the strict inequality $a(x, t)>\alpha$ )

$$
N^{-1} \ln ^{2} N\left(1+\left(1+\alpha h \varepsilon^{-1}\right)^{i+1-N}\right),
$$

with the stability properties of $\hat{L}^{N, M}$ to complete the proof. flux.

Consider now the contribution of the regular component to the discrete error

Lemma 2. Assume (10). For $v, V$, the respective solutions of (15), (11), we have that

$$
\varepsilon\left|D_{x}^{-}(V-v)\left(1, t_{j}\right)\right| \leq C N^{-1}, \quad t_{j} \geq 0 .
$$

Proof. It follows using a similar argument to the proof of Lemma 1 but involving all the grid points of $\bar{G}^{N, M}$. Use the barrier function

$$
B_{2}\left(x_{i}, t_{j}\right):=C\left\|L^{N, M}(V-v)\right\|_{G^{N, M}}\left(x_{i}-1+\tilde{\psi}\left(x_{i}, t_{j}\right)\right),
$$

where

$$
-\varepsilon \delta_{x}^{2} \tilde{\psi}+\left(a\left(x_{i}, t_{j}\right)+\beta t_{j}\right) D_{x}^{-} \tilde{\psi}=0, x_{i} \in(0,1), \quad \tilde{\psi}\left(0, t_{j}\right)=1, \tilde{\psi}\left(1, t_{j}\right)=0 .
$$

Then $\varepsilon\left|\mathscr{V}_{N, j}^{-}\right| \leq C \varepsilon\left\|L^{N, M}(V-v)\right\|_{G^{N, M}}\left(1+\left|D_{x}^{-} \tilde{\psi}\left(1, t_{j}\right)\right|\right) \leq C N^{-1}$.

Theorem 4. Assume (10). Then, for all $t_{j} \geq 0$,

$$
\begin{array}{ll}
\left|D_{x}^{-}(V-v)\left(x_{i}, t_{j}\right)\right| \leq C N^{-1}, & \text { if } x_{i} \leq 1-\sigma, \\
\varepsilon\left|D_{x}^{-}(V-v)\left(x_{i}, t_{j}\right)\right| \leq C N^{-1}(\ln N)^{2}, & \text { if } x_{i}>1-\sigma,
\end{array}
$$

where $V$ is the solution of (11) and $v$ is the solution of (15a).

Proof. We again apply a stability and consistency argument, but now across the domain $G_{H}^{N, M}$ to deduce suitable bounds on $\mathscr{V}^{-}$. At the interior points, using the bounds (14), we get that

$$
\begin{aligned}
&\left|\hat{L}^{N, M} \mathscr{V}_{i, j}^{-}\right| \leq C N^{-1}, \quad x_{i} \neq 1-\sigma, 1-\sigma+h, \\
&\left|\hat{L}^{N, M \mathscr{V}_{i, j}^{-}}\right| \leq C\left(\varepsilon+N^{-1}\right), \quad x_{i}=1-\sigma,
\end{aligned}
$$

and if $x_{i}=1-\sigma+h$, using $(14 \mathrm{a})$, we have that

$$
\left|\hat{L}^{N, M} \mathscr{V}_{i, j}^{-}\right| \leq \frac{C}{h}\left(\varepsilon H\left\|v_{x x x}\right\|_{G}+H\left\|v_{x x}\right\|_{G}\right)+C N^{-1} \leq \frac{C}{\varepsilon \ln N}
$$


Using a suitable barrier function we can establish that $\left|(V-v)\left(x_{i}, t_{j}\right)\right| \leq C x_{i} N^{-1}$ and, hence,

$$
\left|D_{x}^{-}(V-v)\left(x_{1}, t_{j}\right)\right| \leq C \frac{x_{1}}{H} N^{-1} \leq C N^{-1} .
$$

We can deduce appropriate bounds for $\mathscr{V}_{i, j}^{-}$by again constructing a suitable barrier function. Define the following two mesh functions:

$$
R\left(x_{i}\right):=\left\{\begin{array}{ll}
\frac{x_{i}}{1-\sigma}, & \text { if } x_{i} \leq 1-\sigma, \\
1, & \text { if } 1-\sigma<x_{i} \leq 1,
\end{array} \quad S\left(x_{i}\right):= \begin{cases}0, & \text { if } x_{i} \leq 1-\sigma \\
1, & \text { if } 1-\sigma<x_{i} \leq 1 .\end{cases}\right.
$$

Observe that

$$
\hat{L}^{N, M} R\left(x_{i}\right) \geq \begin{cases}\alpha, & \text { if } x_{i}<1-\sigma \\ \varepsilon H^{-1}+\alpha, & \text { if } x_{i}=1-\sigma \\ b+D_{x}^{-} a, & \text { if } x_{i}>1-\sigma\end{cases}
$$

and

$$
\hat{L}^{N, M} S\left(x_{i}\right) \geq \begin{cases}0, & \text { if } x_{i}<1-\sigma \\ -\varepsilon N H^{-1}, & \text { if } x_{i}=1-\sigma \\ \varepsilon N h^{-1}+\alpha h^{-1}, & \text { if } x_{i}=1-\sigma+h \\ b+D_{x}^{-} a, & \text { if } x_{i}>1-\sigma+h .\end{cases}
$$

Define the piecewise linear barrier function

$$
B_{3}\left(x_{i}\right)=C N^{-1} \varepsilon^{-1}\left(R\left(x_{i}\right)+N^{-1} S\left(x_{i}\right)\right)+C N^{-1} R\left(x_{i}\right) .
$$

Then, the discrete maximum principle establishes the bound

$$
\left|\mathscr{V}_{i, j}^{-}\right| \leq C B_{3}\left(x_{i}\right) \leq C N^{-1} \varepsilon^{-1} .
$$

By using a sharper barrier function, we will next remove the scaling factor $\varepsilon$ outside the layer. Define the local mesh Peclet numbers as follows:

$$
\xi:=\frac{\alpha H}{\varepsilon}, \quad \rho:=\frac{\alpha h}{\varepsilon},
$$

and the following two mesh functions:

$$
\begin{aligned}
& P\left(x_{i}\right):= \begin{cases}(1+\xi)^{i-N / 2}, & \text { if } x_{i} \leq 1-\sigma, \\
1, & \text { if } x_{i}=1-\sigma+h, \\
(1+0.5 \rho)^{i-N / 2-1}, & \text { if } 1-\sigma+h<x_{i} \leq 1\end{cases} \\
& Q\left(x_{i}\right):= \begin{cases}0, & \text { if } x_{i} \leq 1-\sigma, \\
(1+0.5 \rho)^{i-N / 2-1}, & \text { if } x_{i}>1-\sigma .\end{cases}
\end{aligned}
$$

Hence, using $2 \varepsilon \ln N \leq 1$ and $N$ sufficiently large,

$$
\hat{L}^{N, M}\left(P\left(x_{i}\right)+\alpha N^{-1} \varepsilon^{-1} Q\left(x_{i}\right)\right) \geq \begin{cases}0, & \text { if } x_{i} \leq 1-\sigma \\ C N \sigma^{-1}, & \text { if } x_{i}=1-\sigma+h \\ C \varepsilon^{-1}, & \text { if } x_{i}>1-\sigma+h\end{cases}
$$

Form the barrier function

$$
B_{4}\left(x_{i}\right):=C N^{-1} R\left(x_{i}\right)+C(P(1))^{-1}\left(P\left(x_{i}\right)+\alpha N^{-1} \varepsilon^{-1} Q\left(x_{i}\right)\right) .
$$

Then we have established that $\left|\mathscr{V}_{i, j}^{-}\left(x_{i}, t_{j}\right)\right| \leq C B_{4}\left(x_{i}\right)$. This bound is of little value in the area $[1-\sigma, 1]\left(\right.$ as $B_{4}\left(x_{i}\right) \leq C\left(1+N^{-1} \varepsilon^{-1}\right)$ in this area). However, in the coarse mesh region $x_{i} \in[0,1-\sigma]$, we deduce that $\left|\mathscr{V}_{i, j}^{-}\right| \leq C B_{4}\left(x_{i}\right) \leq C N^{-1}, x_{i} \leq$ $1-\sigma$. 
Using the bounds obtained for the regular and singular components, the triangular inequality and the truncation error bound

$$
\left\|D_{x}^{-} u-u_{x}\right\|_{I_{i}} \leq C \min \left\{\left\|u_{x}\right\|_{I_{i}}, h_{i}\left\|u_{x x}\right\|_{I_{i}}\right\},
$$

we obtain the following result.

Theorem 5. Assume (10). Then, for all $t_{j} \geq 0$,

$$
\begin{array}{ll}
\left|\left(D_{x}^{-} U-u_{x}\right)\left(x_{i}, t_{j}\right)\right| \leq C N^{-1}, & \text { if } 0<x_{i} \leq 1-\sigma, \\
\varepsilon\left|\left(D_{x}^{-} U-u_{x}\right)\left(x_{i}, t_{j}\right)\right| \leq C N^{-1}(\ln N)^{2}, & \text { if } 1-\sigma<x_{i} \leq 1,
\end{array}
$$

where $U$ is the solution generated by the numerical method (9) and $u$ is the solution of the continuous problem (4).

\section{NodAl APPROXIMATION OF time DERIVATIVES}

We follow the approach outlined in [8, Appendix A.2] and [10, §8.2] to deduce nodal approximations of the time derivatives. We note that the proof in [10] was given only for the case of constant $a$.

Lemma 3. Assume (10). The following bounds hold, for all $t_{j}>0$ :

$$
\begin{array}{ll}
\left|\left(D_{t}^{-} V-v_{t}\right)\left(x_{i}, t_{j}\right)\right| \leq C N^{-1} \ln N, & \text { if } x_{i} \in[0,1], \\
\left|\left(D_{t}^{-} W-w_{t}\right)\left(x_{i}, t_{j}\right)\right| \leq C N^{-1}, & \text { if } x_{i} \in[0,1-\sigma], \\
\left|\left(D_{t}^{-} W-w_{t}\right)\left(x_{i}, t_{j}\right)\right| \leq C N^{-1}(\ln N)^{3}, & \text { if } x_{i} \in[1-\sigma, 1],
\end{array}
$$

where $v, w$ are the solutions of problems (5a), (6a), and $V, W$ are defined in (11).

Proof. Using the bounds on the components $v, w$ of the solution $u$ of problem (4), we deduce that for all $\left(x_{i}, t_{j}\right) \in[0,1] \times[\tau, T]$,

$$
\left|\left(D_{t}^{-} u-u_{t}\right)\left(x_{i}, t_{j}\right)\right| \leq C \tau\left\|u_{t t}\right\| \leq C N^{-1} \text {. }
$$

Hence,

$$
\begin{aligned}
\left|\left(D_{t}^{-} V-v_{t}\right)\left(x_{i}, t_{j}\right)\right| & \leq\left|D_{t}^{-}(V-v)\left(x_{i}, t_{j}\right)\right|+C N^{-1} \\
\left|\left(D_{t}^{-} W-w_{t}\right)\left(x_{i}, t_{j}\right)\right| & \leq\left|D_{t}^{-}(W-w)\left(x_{i}, t_{j}\right)\right|+C N^{-1} .
\end{aligned}
$$

Note that along the side boundaries

$$
D_{t}^{-}(V-v)\left(x_{i}, t_{j}\right)=0, \quad\left(x_{i}, t_{j}\right) \in\left(\Gamma_{L} \cup \Gamma_{R}\right) \cap \bar{G}^{N, M}, \quad t_{j} \geq \tau,
$$

and from the error bound in Theorem 2 we deduce that $\left|D_{t}^{-}(V-v)\left(x_{i}, \tau\right)\right| \leq C N^{-1}$. At the interior points $\left(x_{i}, t_{j}\right) \in G^{N, M} \cap\left\{t_{j} \geq 2 \tau\right\}$, we will first estimate

$$
\left|L^{N, M}\left(D_{t}^{-}(V-v)\left(x_{i}, t_{j}\right)\right)\right| .
$$

We wish to reverse the order of the operators $L^{N, M}$ and $D_{t}^{-}$. To this end, we use again the identity (12) and we define a minor modification to the operator $L^{N, M}$ denoted by

$$
\tilde{L}^{N, M} Z_{i, j}:=\left(L^{N, M}+\left(c\left(t_{j-1}\right)-c\left(t_{j}\right)\right) D_{t}^{-}+D_{t}^{-} c\left(t_{j}\right) I\right) Z_{i, j}
$$


to obtain

$$
\begin{aligned}
\left|\tilde{L}^{N, M} D_{t}^{-}(V-v)\right|= & \mid D_{t}^{-}\left(L^{N, M}(V-v)\right)-D_{t}^{-}(b(V-v))+b D_{t}^{-}(V-v) \\
& -D_{t}^{-}\left(a D_{x}^{-}(V-v)\right)+a D_{x}^{-} D_{t}^{-}(V-v) \mid \\
\leq & \left|D_{t}^{-}\left(L^{N, M}(V-v)\right)\right|+C N^{-1}+C\left|D_{x}^{-}(V-v)\right| \\
\leq & \varepsilon\left|D_{t}^{-}\left(\delta_{x}^{2} v-v_{x x}\right)\right|+C\left|D_{t}^{-}\left(D_{x}^{-} v-v_{x}\right)\right| \\
& +C\left|D_{t}^{-}\left(D_{t}^{-} v-v_{t}\right)\right|+C N^{-1}+C\left|D_{x}^{-}(V-v)\right| \\
\leq & \varepsilon \hbar_{i}\left\|v_{x x x t}\right\|_{G}+C h_{i}\left\|v_{x x t}\right\|_{G}+C\left(\tau\left\|v_{t t}\right\|_{G}+N^{-1}\right) \\
& +C\left\|D_{x}^{-}(V-v)\right\|_{G^{N, M}} \leq C N^{-1}+C\left\|D_{x}^{-}(V-v)\right\|_{G^{N, M}} .
\end{aligned}
$$

We recall that for all $t_{j} \geq 0$,

$$
\left|D_{x}^{-}(V-v)\left(x_{i}, t_{j}\right)\right| \leq \begin{cases}C N^{-1}, & \text { if } x_{i} \leq 1-\sigma \\ C N^{-1} \varepsilon^{-1}, & \text { if } x_{i}>1-\sigma .\end{cases}
$$

Consider the mesh function

$$
Z\left(x_{i}\right):= \begin{cases}(1+\xi)^{i-N / 2}, & \text { if } x_{i} \leq 1-\sigma \\ 1+\left(x_{i}-(1-\sigma)\right) / \sigma, & \text { if } 1-\sigma \leq x_{i} \leq 1\end{cases}
$$

with $\xi=\alpha \varepsilon / H$. Note that

$$
\tilde{L}^{N, M} Z\left(x_{i}\right) \geq 0, x_{i} \leq 1-\sigma, \quad \tilde{L}^{N, M} Z\left(x_{i}\right) \geq \alpha \sigma^{-1}, x_{i}>1-\sigma .
$$

Use the barrier function $C N^{-1}\left(1+Z\left(x_{i}\right) \ln N\right)$ to deduce that

$$
\left|D_{t}^{-}(V-v)\left(x_{i}, t_{j}\right)\right| \leq C N^{-1} \ln N, \quad\left(x_{i}, t_{j}\right) \in[0,1] \times[\tau, T] .
$$

Now we consider the singular component. Outside the layer region, we use the bound

$$
\max \left\{\left|W\left(x_{i}, t_{j}\right)\right|,\left|w\left(x_{i}, t_{j}\right)\right|\right\} \leq C N^{-2}, \quad x_{i} \leq 1-\sigma,
$$

to deduce that, for all $t_{j} \geq \tau$,

$$
\left|D_{t}^{-}(W-w)\left(x_{i}, t_{j}\right)\right| \leq C N^{-1}, \quad x_{i} \leq 1-\sigma .
$$

It now remains to bound $D_{t}^{-}(W-w)$ within the layer region. We repeat the argument from above to deduce the truncation error estimate

$$
\left|\tilde{L}^{N, M}\left(D_{t}^{-}(W-w)\left(x_{i}, t_{j}\right)\right)\right| \leq C \frac{N^{-1}(\ln N)^{2}}{\varepsilon}, x_{i}>1-\sigma, t_{j}>\tau .
$$

At the first time level $t_{j}=\tau$, using the bounds in Theorem 2 we deduce that

$$
\left|\left(D_{t}^{-}(W-w)\left(x_{i}, \tau\right)\right)\right| \leq C N^{-1} \ln N, x_{i}>1-\sigma,
$$

and at the right boundary, $D_{t}^{-}(W-w)\left(1, t_{j}\right)=0$. Use the discrete barrier function

$$
C\left(\frac{x_{i}-(1-\sigma)}{\varepsilon} N^{-1}(\ln N)^{2}+N^{-1} \ln N\right)
$$

to complete the proof.

Motivated by the bounds in [12, Corollary 3] we can sharpen the bound given in Theorem 5 for points within the layer region in the special case where $N^{-2} \leq \varepsilon$.

Theorem 6. Assume (10). If $N^{-2} \leq \varepsilon$, then

$$
\left|D_{x}^{-}(U-u)\left(x_{i}, t_{j}\right)\right| \leq C N^{-1}(\ln N)^{3}, \quad x_{i}<1-\frac{\varepsilon}{\alpha} \ln \frac{1}{\varepsilon}, t_{j} \geq 0,
$$

where $U, u$ are the respective solutions of (9) and (4). 
Proof. If $N^{-2} \leq \varepsilon$, then $\varepsilon \ln \frac{1}{\varepsilon} \leq 2 \varepsilon \ln N$. From the previous result, we only need to consider the mesh points in the region $\left(1-2 \frac{\varepsilon}{\alpha} \ln N, 1-\frac{\varepsilon}{\alpha} \ln \frac{1}{\varepsilon}\right)$. Within the fine mesh, the error satisfies

$$
-\frac{\varepsilon}{h}\left(\mathscr{U}_{i+1, j}^{-}-\mathscr{U}_{i, j}^{-}\right)+a\left(x_{i}, t_{j}\right) \mathscr{U}_{i, j}^{-}=\hat{\mathscr{T}}_{i, j},
$$

where $\hat{\mathscr{T}}_{i, j}=L^{N, M}(U-u)\left(x_{i}, t_{j}\right)-c\left(t_{j}\right) D_{t}^{-}(U-u)\left(x_{i}, t_{j}\right)-b\left(x_{i}, t_{j}\right)(U-u)\left(x_{i}, t_{j}\right)$. Note that

$$
\|\hat{\mathscr{T}}\|_{G_{0}^{N, M}} \leq C\left(N^{-1} \ln N \varepsilon^{-1} e^{-\alpha\left(1-x_{i}\right) / \varepsilon}+N^{-1}(\ln N)^{3},\right.
$$

with $G_{0}^{N, M}=\bar{G}^{N, M} \cap\left(1-2 \frac{\varepsilon}{\alpha} \ln N, 1-\frac{\varepsilon}{\alpha} \ln \frac{1}{\varepsilon}\right)$. Thus, with $\rho:=\frac{\alpha h}{\varepsilon}$, we have

$$
\left|\mathscr{U}_{i, j}^{-}\right|=\left(1+\frac{h}{\varepsilon} a\left(x_{i}, t_{j}\right)\right)^{-1}\left|\frac{h}{\varepsilon} \hat{\mathscr{T}}_{i, j}+\mathscr{U}_{i+1, j}^{-}\right| \leq(1+\rho)^{-1}\left(\frac{h}{\varepsilon}\|\hat{\mathscr{T}}\|_{G_{0}^{N, M}}+\left|\mathscr{U}_{i+1, j}^{-}\right|\right) .
$$

Thus, we have the following estimate at $x_{i}$ (within the fine mesh):

$$
\begin{aligned}
\left|\mathscr{U}_{i, j}^{-}\right| & \leq(1+\rho)^{-1} \frac{h}{\varepsilon}\|\hat{\mathscr{T}}\|_{G_{0}^{N, M}} \frac{1-(1+\rho)^{-(N-i)}}{1-(1+\rho)^{-1}}+C(1+\rho)^{-(N-i)}\left|\mathscr{U}_{N, j}^{-}\right| \\
& \leq C\|\hat{\mathscr{T}}\|_{G_{0}^{N, M}}+C \frac{N^{-1}(\ln N)^{2}}{\varepsilon}(1+\rho)^{-(N-i)} .
\end{aligned}
$$

Since $x_{i}<1-\frac{\varepsilon}{\alpha} \ln \frac{1}{\varepsilon}$, there exists some $\theta>1$ such that $x_{i} \leq 1-\theta \frac{\varepsilon}{\alpha} \ln \frac{1}{\varepsilon}$. For $N$ sufficiently large, we note that, $(1+\rho)^{-1} \leq e^{-\rho / \theta}, \rho \leq \theta \ln \theta, \theta>1$. Hence, for $x_{i} \leq 1-\theta \frac{\varepsilon}{\alpha} \ln \frac{1}{\varepsilon}$,

$$
\left|\mathscr{U}_{i, j}^{-}\right| \leq C\left(N^{-1}(\ln N)^{2} \varepsilon^{-1} e^{-\frac{\alpha\left(1-x_{i}\right)}{\theta \varepsilon}}+N^{-1}(\ln N)^{3}\right) \leq C N^{-1}(\ln N)^{3} .
$$

\section{Global aCCURACY in weighted $C^{1}$-NORM}

In this section, we examine the global accuracy (in the weighted $C^{1}$-norm $\left.\|\cdot\|_{1, \chi, G}\right)$ of the bilinear interpolant

$$
\bar{U}(x, t):=\sum_{i, j=1}^{N-1, M} U\left(x_{i}, t_{j}\right) \phi_{i}(x) \psi_{j}(t), \quad(x, t) \in \bar{G},
$$

where $\phi_{i}(x), \psi_{j}(t)$ are piecewise linear basis functions in space and time, defined by the nodal values of $\phi_{i}\left(x_{k}\right)=\delta_{i, k}=\psi_{i}\left(t_{k}\right)$. Note the following bound on the bilinear interpolant $\bar{g}$ of a function $g$ (see e.g. [16, Lemma 4.1]) in the rectangular cell $R_{i j}:=\left(x_{i-1}, x_{i}\right) \times\left(t_{j-1}, t_{j}\right)$ :

$$
\begin{aligned}
\|g-\bar{g}\|_{R_{i j}} \leq & C \min \left\{h_{i}^{2}\left\|g_{x x}\right\|_{R_{i j}}, \max _{t \in\left[t_{j-1}, t_{j}\right]} \int_{x_{i-1}}^{x_{i}}\left|g_{x}(s, t)\right| d s\right\} \\
& +C \min \left\{\tau^{2}\left\|g_{t t}\right\|_{R_{i j}}, \max _{x \in\left[x_{i-1}, x_{i}\right]} \int_{t_{j-1}}^{t_{j}}\left|g_{t}(x, s)\right| d s\right\} .
\end{aligned}
$$

Theorem 7. Assume (10). Then,

$$
\|\bar{U}-u\|_{1, \chi, G} \leq C N^{-1}(\ln N)^{3},
$$

where $U$ is the solution generated by the numerical method (9) and $u$ is the solution of the continuous problem (4). 
Proof. Using the decomposition $u=v+w$ and splitting the argument to inside and outside the computational layer region $[1-\sigma, 1] \times(0, T)$, we have the following interpolation error (see e.g. [16, Theorem 4.2]):

$$
\|u-\bar{u}\|_{G} \leq C\left(N^{-1} \ln N\right)^{2}+C \tau^{2} .
$$

Hence, the following global error estimate follows:

$$
\|u-\bar{U}\|_{G} \leq C N^{-1} \ln N .
$$

Note that

$$
\begin{aligned}
& (\bar{U}-\bar{u})_{t}(x, t)=\sum_{i=1}^{N-1} D_{t}^{-}(U-u)\left(x_{i}, t_{j}\right) \phi_{i}(x), \quad t \in\left(t_{j-1}, t_{j}\right] \\
& (\bar{U}-\bar{u})_{x}(x, t)=\sum_{j=1}^{M} D_{x}^{-}(U-u)\left(x_{i}, t_{j}\right) \psi_{j}(t), \quad x \in\left(x_{i-1}, x_{i}\right] .
\end{aligned}
$$

Using the bounds in Lemma 3 for the discrete time derivatives, the bounds in Theorem 5 when $\varepsilon \leq N^{-2}$ and the bound in Theorem 6 when $\varepsilon \geq N^{-2}$ for the discrete space derivatives, we have that

$$
\|\bar{U}-\bar{u}\|_{1, \chi, G} \leq C N^{-1}(\ln N)^{3} .
$$

We are left to estimate the interpolation error $\|u-\bar{u}\|_{1, \chi, G}$. For $x \in\left(x_{i-1}, x_{i}\right]$, we have

$$
(\bar{u}-u)_{x}(x, t)=\sum_{j=1}^{M}\left(D_{x}^{-} u\left(x_{i}, t_{j}\right)-u_{x}\left(x, t_{j}\right)\right) \psi_{j}(t)+\sum_{j=1}^{M} u_{x}\left(x, t_{j}\right) \psi_{j}(t)-u_{x}(x, t) .
$$

Therefore, in the rectangular cell $R_{i j}$, we obtain

$$
\left\|(g-\bar{g})_{x}\right\|_{R_{i j}} \leq \min \left\{h_{i}\left\|g_{x x}\right\|_{R_{i j}},\left\|g_{x}\right\|_{R_{i j}}\right\}+\min \left\{\tau\left\|g_{x t}\right\|_{R_{i j}},\left\|g_{x}\right\|_{R_{i j}}\right\} .
$$

We employ the decomposition $u=v+w$. For the regular component it trivially follows that

$$
\left\|(v-\bar{v})_{x}\right\|_{R_{i j}} \leq C\left(N^{-1}+\tau\right) .
$$

For the layer component, we split the argument to inside and outside the layer region $\left[1-2 \frac{\varepsilon}{\alpha} \ln \frac{1}{\varepsilon}, 1\right] \times(0, T]$ and deal with the two cases of $\varepsilon \leq N^{-2}$ and $\varepsilon \geq N^{-2}$. We observe the following: If $\varepsilon \leq N^{-2}$, then $2 \varepsilon \ln N \leq \varepsilon \ln \frac{1}{\varepsilon}$ and in this case

$$
\begin{aligned}
\left\|w_{x}\right\|_{R_{i j}} & \leq C \varepsilon \leq C N^{-2}, \quad \text { if } x_{i} \leq 1-2(\varepsilon / \alpha) \ln (1 / \varepsilon), \\
\left\|w_{x}\right\|_{R_{i j}} & \leq C \varepsilon^{-1} N^{-2}, \quad \text { if } 1-2(\varepsilon / \alpha) \ln (1 / \varepsilon)<x_{i} \leq 1-\sigma, \\
h_{i}\left\|w_{x x}\right\|_{R_{i j}}+\tau\left\|w_{x t}\right\|_{R_{i j}} & \leq C \varepsilon^{-1} N^{-1} \ln N, \quad \text { if } x_{i}>1-\sigma .
\end{aligned}
$$

In the second case, where $\varepsilon \geq N^{-2}$, we then distinguish two subcases: If $\varepsilon \geq N^{-1}$, then

$$
\begin{aligned}
& h_{i}\left\|w_{x x}\right\|_{R_{i j}}+\tau\left\|w_{x t}\right\|_{R_{i j}} \leq C\left(h_{i}+\tau\right), \quad \text { if } x_{i} \leq 1-2(\varepsilon / \alpha) \ln (1 / \varepsilon), \\
& h_{i}\left\|w_{x x}\right\|_{R_{i j}}+\tau\left\|w_{x t}\right\|_{R_{i j}} \leq C \varepsilon^{-1} N^{-1} \ln N, \quad \text { if } x_{i}>1-2(\varepsilon / \alpha) \ln (1 / \varepsilon) .
\end{aligned}
$$

On the other hand, if $N^{-1} \geq \varepsilon \geq N^{-2}$, then

$$
\begin{aligned}
h_{i}\left\|w_{x x}\right\|_{R_{i j}}+\tau\left\|w_{x t}\right\|_{R_{i j}} & \leq C\left(h_{i}+\tau\right), \quad \text { if } x_{i} \leq 1-2(\varepsilon / \alpha) \ln (1 / \varepsilon), \\
\left\|w_{x}\right\|_{R_{i j}} & \leq C \varepsilon^{-1} N^{-2}, \quad \text { if } 1-2(\varepsilon / \alpha) \ln (1 / \varepsilon)<x_{i} \leq 1-\sigma, \\
h_{i}\left\|w_{x x}\right\|_{R_{i j}}+\tau\left\|w_{x t}\right\|_{R_{i j}} & \leq C \varepsilon^{-1} N^{-1} \ln N, \quad \text { if } x_{i}>1-\sigma .
\end{aligned}
$$


Combining all these bounds, we deduce that

$$
\left\|\chi(x)(u-\bar{u})_{x}\right\|_{G} \leq C N^{-1} \ln N,
$$

where $\chi(x)$ is defined in (3). Similarly, for $t \in\left(t_{j}-1, t_{j}\right]$,

$$
(\bar{u}-u)_{t}(x, t)=\sum_{i=1}^{N-1}\left(D_{t}^{-} u\left(x_{i}, t_{j}\right)-u_{t}\left(x_{i}, t\right)\right) \phi_{i}(x)+\sum_{i=1}^{N-1} u_{t}\left(x_{i}, t\right) \phi_{i}(x)-u_{t}(x, t) .
$$

In the rectangular cell $R_{i j}$,

$$
\left\|(g-\bar{g})_{t}\right\|_{R_{i j}} \leq C \tau\left\|g_{t t}\right\|_{R_{i j}}+\min \left\{h_{i}\left\|g_{x t}\right\|_{R_{i j}},\left\|g_{t}\right\|_{R_{i j}}\right\} .
$$

By again using the decomposition $u=v+w$ and splitting the argument to inside and outside the layer region $\left[1-2 \frac{\varepsilon}{\alpha} \ln \frac{1}{\varepsilon}, 1\right] \times(0, T]$, we deduce that $\left\|(u-\bar{u})_{t}\right\|_{G} \leq$ $C N^{-1}$.

Remark 3. The bound (21) in Theorem 7 can be easily extended to the classical case of $\sigma=0.5$ by using the inequality $\varepsilon^{-1} \leq C \ln N$ when $\sigma=0.5$.

\section{NUMERICAL EXPERIMENTS}

In this section we consider the variable coefficient problem

$$
\begin{array}{r}
-\varepsilon u_{x x}+\left(1+x+4 t^{2}\right) u_{x}+x u+u_{t}=50 x^{4} t^{2}(1-x)^{2}, \\
u(0, t)=u(1, t)=0, u(x, 0)=(4 x(1-x))^{3},
\end{array}
$$

whose solution is not explicitly known.

To estimate the errors in the norm $\|\cdot\|_{1, \chi, G}$ of the numerical scheme (9) for any fixed value of the singular perturbation parameter $\varepsilon$, we use a variant of the double mesh principle (see [5]): Given a numerical approximation $U^{N, M}$ generated over a mesh $\bar{G}^{N, M}$, we also generate the numerical solution on a fine Shishkin mesh $U^{2048,2048}$ with $N, M<2048$, and compute the global fine mesh differences:

$$
E_{\varepsilon}^{N, M}:=\max \left\{\left\|U^{N, M}-\bar{U}^{2048,2048}\right\|_{1, \chi, \bar{G}^{N, M}},\left\|\bar{U}^{N, M}-U^{2048,2048}\right\|_{1, \chi, \bar{G}^{2048,2048}}\right\},
$$

where $\bar{U}^{N, M}$ denotes the bilinear interpolant of the numerical solution over the mesh $\bar{G}^{N, M}$. From these values, we compute the approximate order of convergence using

$$
Q_{\varepsilon}^{N, M}:=\log _{2}\left(E_{\varepsilon}^{N, M} / E_{\varepsilon}^{2 N, 2 M}\right) .
$$

The uniform global errors in the norm $\|\cdot\|_{1, \chi, G}$ and their orders of convergence are estimated as follows:

$$
E^{N, M}:=\max _{\varepsilon \in S} E_{\varepsilon}^{N, M}, \quad Q^{N, M}:=\log _{2}\left(E^{N, M} / E^{2 N, 2 M}\right),
$$

with $S=\left\{2^{0}, 2^{-1}, 2^{-2}, \ldots, 2^{-30}\right\}$.

In Table 1 we present the global $E_{\varepsilon}^{N, M}$ and uniform global $E^{N, M}$ computed errors for $N=M=2^{j}, j=4,5,6,7,8,9$, with their corresponding orders of convergence associated with the finite difference scheme (9) on the Shishkin mesh for test problem (22). The numerical results in Table 1 indicate that the method is uniformly convergent in the weighted $C^{1}$-norm $\|\cdot\|_{1, \chi, G}$. The computed orders of convergence in this example are slightly higher than the theoretical order of convergence established in Theorem 7 , but this is a well-known effect when the errors are estimated by considering the computed solution on a fine mesh as the exact solution. 
TABLE 1. Finite difference scheme (9) on the Shishkin mesh: Global computed errors in $\|\cdot\|_{1, \chi, G}$ estimated by $E_{\varepsilon}^{N, M}$ and uniform global computed errors $E^{N, M}$ with their corresponding computed orders of convergence $Q_{\varepsilon}^{N, M}, Q^{N, M}$ for the test problem (22).

\begin{tabular}{|c|c|c|c|c|c|c|}
\hline & $\mathrm{N}=\mathrm{M}=16$ & $\mathrm{~N}=\mathrm{M}=32$ & $\mathrm{~N}=\mathrm{M}=64$ & $\mathrm{~N}=\mathrm{M}=128$ & $\mathrm{~N}=\mathrm{M}=256$ & $\mathrm{~N}=\mathrm{M}=512$ \\
\hline$\varepsilon=2^{0}$ & $\begin{array}{c}2.040 \mathrm{E}+001 \\
0.216\end{array}$ & $\begin{array}{c}1.757 \mathrm{E}+001 \\
0.360\end{array}$ & $\begin{array}{c}1.369 \mathrm{E}+001 \\
0.540\end{array}$ & $\begin{array}{c}9.412 \mathrm{E}+000 \\
0.703\end{array}$ & $\begin{array}{c}5.781 \mathrm{E}+000 \\
0.867\end{array}$ & $3.170 \mathrm{E}+000$ \\
\hline$\varepsilon=2^{-1}$ & $\begin{array}{c}1.090 \mathrm{E}+001 \\
0.415\end{array}$ & $\begin{array}{c}8.177 \mathrm{E}+000 \\
0.594\end{array}$ & $\begin{array}{c}5.417 \mathrm{E}+000 \\
0.702\end{array}$ & $\begin{array}{c}3.329 \mathrm{E}+000 \\
0.708\end{array}$ & $\begin{array}{c}2.038 \mathrm{E}+000 \\
0.933\end{array}$ & $1.067 \mathrm{E}+000$ \\
\hline$\varepsilon=2^{-2}$ & $\begin{array}{c}6.162 \mathrm{E}+000 \\
0.650 \\
\end{array}$ & $\begin{array}{c}3.928 \mathrm{E}+000 \\
0.817 \\
\end{array}$ & $\begin{array}{c}2.230 \mathrm{E}+000 \\
0.848 \\
\end{array}$ & $\begin{array}{c}1.239 \mathrm{E}+000 \\
0.763 \\
\end{array}$ & $\begin{array}{c}7.300 \mathrm{E}-001 \\
0.973 \\
\end{array}$ & $3.720 \mathrm{E}-001$ \\
\hline$\varepsilon=2^{-3}$ & $\begin{array}{c}4.678 \mathrm{E}+000 \\
0.742\end{array}$ & $\begin{array}{c}2.796 \mathrm{E}+000 \\
0.886\end{array}$ & $\begin{array}{c}.513 \mathrm{E}+000 \\
0.978\end{array}$ & $\begin{array}{c}7.684 \mathrm{E}-001 \\
1.068\end{array}$ & $\begin{array}{c}3.665 \mathrm{E}-001 \\
1.207\end{array}$ & $1.588 \mathrm{E}-001$ \\
\hline$\varepsilon=2^{-4}$ & $\begin{array}{c}4.681 \mathrm{E}+000 \\
0.859 \\
\end{array}$ & $\begin{array}{c}2.580 \mathrm{E}+000 \\
0.929 \\
\end{array}$ & $\begin{array}{c}1.355 \mathrm{E}+000 \\
0.875 \\
\end{array}$ & $\begin{array}{c}7.390 \mathrm{E}-001 \\
0.993 \\
\end{array}$ & $\begin{array}{c}3.712 \mathrm{E}-001 \\
1.165 \\
\end{array}$ & $1.656 \mathrm{E}-001$ \\
\hline$\varepsilon=2^{-5}$ & $\begin{array}{c}5.164 \mathrm{E}+000 \\
0.810 \\
\end{array}$ & $\begin{array}{c}2.946 \mathrm{E}+000 \\
0.808 \\
\end{array}$ & $\begin{array}{c}1.683 \mathrm{E}+000 \\
0.789 \\
\end{array}$ & $\begin{array}{c}9.742 \mathrm{E}-001 \\
0.897\end{array}$ & $\begin{array}{c}.231 \mathrm{E}-001 \\
1.077 \\
\end{array}$ & $2.480 \mathrm{E}-001$ \\
\hline$\varepsilon=2^{-6}$ & $\begin{array}{c}5.393 \mathrm{E}+000 \\
0.627\end{array}$ & $\begin{array}{c}3.492 \mathrm{E}+000 \\
0.727\end{array}$ & $\begin{array}{c}2.110 \mathrm{E}+000 \\
0.756\end{array}$ & $\begin{array}{c}1.249 \mathrm{E}+000 \\
0.910\end{array}$ & $\begin{array}{c}6.649 \mathrm{E}-001 \\
1.095 \\
\end{array}$ & $3.113 \mathrm{E}-001$ \\
\hline$\varepsilon=2^{-7}$ & $\begin{array}{c}5.685 \mathrm{E}+000 \\
0.551 \\
\end{array}$ & $\begin{array}{c}3.881 \mathrm{E}+000 \\
0.677\end{array}$ & $\begin{array}{c}2.427 \mathrm{E}+000 \\
0.769 \\
\end{array}$ & $\begin{array}{c}1.425 \mathrm{E}+000 \\
0.899\end{array}$ & $\begin{array}{c}7.643 \mathrm{E}-001 \\
1.091 \\
\end{array}$ & $3.587 \mathrm{E}-001$ \\
\hline$\varepsilon=2^{-8}$ & $\begin{array}{c}5.850 \mathrm{E}+000 \\
0.517\end{array}$ & $\begin{array}{c}4.087 \mathrm{E}+000 \\
0.606\end{array}$ & $\begin{array}{c}2.685 \mathrm{E}+000 \\
0.767\end{array}$ & $\begin{array}{c}1.578 \mathrm{E}+000 \\
0.935\end{array}$ & $\begin{array}{c}8.256 \mathrm{E}-001 \\
1.107 \\
\end{array}$ & $3.834 \mathrm{E}-001$ \\
\hline$\varepsilon=2^{-9}$ & $\begin{array}{c}5.978 \mathrm{E}+000 \\
0.513 \\
\end{array}$ & $\begin{array}{c}4.190 \mathrm{E}+000 \\
0.573\end{array}$ & $\begin{array}{c}2.816 \mathrm{E}+000 \\
0.754\end{array}$ & $\begin{array}{c}1.670 \mathrm{E}+000 \\
0.927\end{array}$ & $\begin{array}{c}8.783 \mathrm{E}-001 \\
1.128 \\
\end{array}$ & $4.020 \mathrm{E}-001$ \\
\hline$\varepsilon=2^{-10}$ & $\begin{array}{c}6.047 \mathrm{E}+000 \\
0.501\end{array}$ & $\begin{array}{c}4.273 \mathrm{E}+000 \\
0.569\end{array}$ & $\begin{array}{c}2.881 \mathrm{E}+000 \\
0.748\end{array}$ & $\begin{array}{c}1.715 \mathrm{E}+000 \\
0.924\end{array}$ & $\begin{array}{c}9.042 \mathrm{E}-001 \\
1.123\end{array}$ & $4.152 \mathrm{E}-001$ \\
\hline$\varepsilon=2^{-11}$ & $\begin{array}{c}6.075 \mathrm{E}+000 \\
0.494\end{array}$ & $\begin{array}{c}4.315 \mathrm{E}+000 \\
0.567\end{array}$ & $\begin{array}{c}2.911 \mathrm{E}+000 \\
0.743\end{array}$ & $\begin{array}{c}1.740 \mathrm{E}+000 \\
0.923\end{array}$ & $\begin{array}{c}9.176 \mathrm{E}-001 \\
1.119 \\
\end{array}$ & $4.224 \mathrm{E}-001$ \\
\hline$\varepsilon=2^{-12}$ & $\begin{array}{c}6.089 \mathrm{E}+000 \\
0.490\end{array}$ & $\begin{array}{c}4.336 \mathrm{E}+000 \\
0.566\end{array}$ & $\begin{array}{c}2.928 \mathrm{E}+000 \\
0.741\end{array}$ & $\begin{array}{c}1.752 \mathrm{E}+000 \\
0.922\end{array}$ & $\begin{array}{c}9.245 \mathrm{E}-001 \\
1.118\end{array}$ & $4.261 \mathrm{E}-001$ \\
\hline$\varepsilon=2^{-13}$ & $\begin{array}{c}6.099 \mathrm{E}+000 \\
0.489 \\
\end{array}$ & $\begin{array}{c}4.346 \mathrm{E}+000 \\
0.566 \\
\end{array}$ & $\begin{array}{c}2.936 \mathrm{E}+000 \\
0.740 \\
\end{array}$ & $\begin{array}{c}1.758 \mathrm{E}+000 \\
0.921 \\
\end{array}$ & $\begin{array}{c}9.286 \mathrm{E}-001 \\
1.118 \\
\end{array}$ & $4.278 \mathrm{E}-001$ \\
\hline$\varepsilon=2^{-14}$ & $\begin{array}{c}6.101 \mathrm{E}+000 \\
0.488\end{array}$ & $\begin{array}{c}4.351 \mathrm{E}+000 \\
0.566\end{array}$ & $\begin{array}{c}2.939 \mathrm{E}+000 \\
0.739\end{array}$ & $\begin{array}{c}1.761 \mathrm{E}+000 \\
0.921\end{array}$ & $\begin{array}{c}9.300 \mathrm{E}-001 \\
1.117\end{array}$ & $4.287 \mathrm{E}-001$ \\
\hline & : & : & : & : & : & : \\
\hline$\varepsilon=2^{-29}$ & $\begin{array}{c}6.103 \mathrm{E}+000 \\
0.487\end{array}$ & $\begin{array}{c}4.355 \mathrm{E}+000 \\
0.565\end{array}$ & $\begin{array}{c}2.943 \mathrm{E}+000 \\
0.739\end{array}$ & $\begin{array}{c}1.764 \mathrm{E}+000 \\
0.921\end{array}$ & $\begin{array}{c}9.314 \mathrm{E}-001 \\
1.116\end{array}$ & $4.296 \mathrm{E}-001$ \\
\hline$\varepsilon=2^{-30}$ & $\begin{array}{c}6.103 \mathrm{E}+000 \\
0.487 \\
\end{array}$ & $\begin{array}{c}4.355 \mathrm{E}+000 \\
0.565 \\
\end{array}$ & $\begin{array}{c}2.943 \mathrm{E}+000 \\
0.739 \\
\end{array}$ & $\begin{array}{c}1.763 \mathrm{E}+000 \\
0.921\end{array}$ & $\begin{array}{c}9.314 \mathrm{E}-001 \\
1.116\end{array}$ & $4.296 \mathrm{E}-001$ \\
\hline $\begin{array}{l}E^{N, M} \\
Q^{N, M}\end{array}$ & $\begin{array}{c}2.040 \mathrm{E}+001 \\
0.216\end{array}$ & $\begin{array}{c}1.757 \mathrm{E}+001 \\
0.360\end{array}$ & $\begin{array}{c}1.369 \mathrm{E}+001 \\
0.540\end{array}$ & $\begin{array}{c}9.412 \mathrm{E}+000 \\
0.703\end{array}$ & $\begin{array}{c}5.781 \mathrm{E}+000 \\
0.867\end{array}$ & $3.170 \mathrm{E}+000$ \\
\hline
\end{tabular}

\section{ACKNOWLEDGMENTS}

This research was partially supported by the project MEC/FEDER MTM 201016917 and the Diputación General de Aragón.

\section{REFERENCES}

[1] V. B. Andreev, Pointwise and weighted a priori estimates for the solution and its first derivative of a singularly perturbed convection-diffusion equation (Russian, with Russian summary), Differ. Uravn. 38 (2002), no. 7, 918-929, 1005, DOI 10.1023/A:1021103512850; English transl., Differ. Equ. 38 (2002), no. 7, 972-984. MR2005755 (2004g:34098)

[2] V. B. Andreev, The Green function and a priori estimates for solutions of monotone three-point singularly perturbed difference schemes (Russian, with Russian summary), Differ. Uravn. 37 (2001), no. 7, 880-890, 1005, DOI 10.1023/A:1011949419389; English transl., Differ. Equ. 37 (2001), no. 7, 923-933. MR1887263 (2003f:65132)

[3] V. B. Andreev and I. A. Savin, On the computation of a boundary flux with uniform accuracy with respect to a small parameter (Russian, with Russian summary), Zh. Vychisl. Mat. i Mat. Fiz. 36 (1996), no. 12, 57-63; English transl., Comput. Math. Math. Phys. 36 (1996), no. 12, 1687-1692 (1997). MR1431731 (97m:34029) 
[4] N. S. Bahvalov, On the optimization of the methods for solving boundary value problems in the presence of a boundary layer (Russian), Ž. Vyčisl. Mat. i Mat. Fiz. 9 (1969), 841-859. MR.0255066 (40 \#8273)

[5] P. A. Farrell, A. F. Hegarty, J. J. H. Miller, E. O'Riordan, and G. I. Shishkin, Robust Computational Techniques for Boundary Layers, Applied Mathematics (Boca Raton), vol. 16, Chapman \& Hall/CRC, Boca Raton, FL, 2000. MR1750671 (2001c:65003)

[6] A. Friedman, Partial Differential Equations of Parabolic Type, Prentice-Hall, Inc., Englewood Cliffs, NJ, 1964. MR0181836 (31 \#6062)

[7] E. C. Gartland Jr., Graded-mesh difference schemes for singularly perturbed two-point boundary value problems, Math. Comp. 51 (1988), no. 184, 631-657, DOI 10.2307/2008767. MR.935072(89d:65073)

[8] P. W. Hemker, G. I. Shishkin, and L. P. Shishkina, $\epsilon$-uniform schemes with high-order timeaccuracy for parabolic singular perturbation problems, IMA J. Numer. Anal. 20 (2000), no. 1, 99-121, DOI 10.1093/imanum/20.1.99. MR1736952(2000k:65139)

[9] P. W. Hemker, G. I. Shishkin, and L. P. Shishkina, High-order time-accurate parallel schemes for parabolic singularly perturbed problems with convection, Archives for scientific computing. Numerical methods for transport-dominated and related problems (Magdeburg, 1999), Computing 66 (2001), no. 2, 139-161, DOI 10.1007/s006070170032. MR.1825799(2002d:65088)

[10] P. W. Hemker, G. I. Shishkin, and L. P. Shishkina, High-order time-accuracy schemes for parabolic singular perturbation problems with convection, Russian J. Numer. Anal. Math. Modelling 17 (2002), no. 1, 1-24. MR1890354 (2003g:65100)

[11] N. V. Kopteva, On the convergence, uniform with respect to a small parameter, of a scheme with weights for a one-dimensional nonstationary convection-diffusion equation (Russian, with Russian summary), Zh. Vychisl. Mat. Mat. Fiz. 37 (1997), no. 10, 1213-1220; English transl., Comput. Math. Math. Phys. 37 (1997), no. 10, 1173-1180. MR1481149 (98f:76054)

[12] N. Kopteva and M. Stynes, Approximation of derivatives in a convection-diffusion two-point boundary value problem, Appl. Numer. Math. 39 (2001), no. 1, 47-60, DOI 10.1016/S01689274(01)00051-4. MR.1855164(2002f:65104)

[13] O. A. Ladyženskaja, V. A. Solonnikov, and N. N. Ural'ceva, Linear and Quasilinear Equations of Parabolic Type (Russian), Translated from the Russian by S. Smith. Translations of Mathematical Monographs, Vol. 23, American Mathematical Society, Providence, RI, 1968. MR0241822(39 \#3159b)

[14] G. I. Shishkin, Approximation of solutions and derivatives for singularly perturbed elliptic convection-diffusion equations, Math. Proc. R. Ir. Acad. 103A (2003), no. 2, 169-201, DOI 10.3318/PRIA.2003.103.2.169. MR2138406(2006a:65145)

[15] G. I. Shishkin, Discrete approximations of solutions and derivatives for a singularly perturbed parabolic convection-diffusion equation, Proceedings of the International Conference on Boundary and Interior Layers - Computational and Asymptotic Methods (BAIL 2002), J. Comput. Appl. Math. 166 (2004), no. 1, 247-266, DOI 10.1016/j.cam.2003.09.023. MR2057976 (2005f:65109)

[16] M. Stynes and E. O'Riordan, A uniformly convergent Galerkin method on a Shishkin mesh for a convection-diffusion problem, J. Math. Anal. Appl. 214 (1997), no. 1, 36-54, DOI 10.1006/jmaa.1997.5581. MR.1645503 (99f:65177)

IUMA - Department of Applied Mathematics, University of Zaragoza, 50018 Zaragoza, SPAIN

E-mail address: jlgracia@unizar.es

School of Mathematical Sciences, Dublin City University, Dublin 9, Ireland

E-mail address: eugene.oriordan@dcu.ie 